\title{
Review
}

\section{Determinants of efficacy and toxicity of aminoglycosides}

\author{
H. Mattie, W. A. Craigh and J. C. Pechèree \\ -University Hospital, Department of Infectious Diseases, Bldg 1, C5-P, PO Box 9600, \\ 2300 RC Leiden. The Netherlands; 'William S. Middleton Memorial Veterans' \\ Hospital, 2500 Overlook Terrace, Madison WI 53705, USA; 'Department of \\ Microbiology, Centre Médicale Universitaire, 9, Avenue de Champel, 1211 Geneva, \\ Switzerland
}

The relative efficacy of different aminoglycosides or of different dosage schedules of the same aminoglycoside should be quantitated and related to relative toxicity. Quantitative experimental indicators of efficacy should not only include MIC and $M B C$, but also the postantibiotic effect in vitro and in vivo, the emergence of resistance in in-vitro and in-vivo models, and the relationship between plasma concentration profiles and efficacy. Parameters of clinical efficacy are to be related to pharmacokinetic parameters such as the ratio between the peak serum concentration and the MIC.

Toxicity in clinical trials should be assessed by the most sensitive methods available. Experimental and clinical studies have shown cortical uptake to be a sensitive indicator of renal toxicity. As far as ototoxicity is concerned endolymph and perilymph pharmacokinetics are not clearly related. Clinical ototoxicity should be assessed by sensitive methods, such as high frequency tone audiometry. Finally, risk factors for nephrotoxicity and ototoxicity (e.g., duration of treatment, associated nephrotoxic drugs, dehydration) should be assessed in the evaluation of clinical trials.

\section{Introduction}

As there seems to be no qualitative difference between the various aminoglycosides with respect to their antibacterial efficacy, much emphasis is laid on their relative toxicity when it comes to the choice of a particular aminoglycoside. However, the criteria to be used to compare quantitatively the toxic features of aminoglycosides, especially under clinical conditions, have not been established systematically. Moreover, if quantified at all, they should be evaluated in relation to the efficacy. This review is intended to discuss accepted methods to quantify efficacy and toxicity, in a way to optimize the

Based on contributions to a Wortshop on Aminoglycoside Toxicity, 15-16 May, 1986, Noordwijk ann Zee, The Netherlands, by: J. Aran, Hópital Pelegrin, Bordeaux, France; Ph. Van der Auwera, Institut Jules Bordet, Brussels, Belgium; J. Blaser, Universitatsspital Zurich, Zirich, Switzerland; G. Bongrerts, Sint Radboudziekenhuis, Nijmegen, The Netherlands, W. A Craig, William S. Middleton Memorial Veterans' Hospital, Wisconsin, USA; H. Mattie, Academisch Ziekenhuis, Leiden, The Netherlands; J. C. Pechere, Centre Médicale Universitaire, Geneva, Switzerland; R. A. Tange, Academisch Medisch Centrum, Amsterdam. The Netherlands; P. Tran Ba Huy, Hôpital Lariboisière, Paris, France; P. Tulkens, Université Catholique de Louvain, Brussels, Belgium; G. A. Verpooten, Universitair Ziekenhuis, Antwerpen, Belgium. 
proper choice of an aminoglycoside. It is concluded that data are still insufficient to allow for a definite choice. For instance, any modification in the dose or dosage regimen of a drug that lessens toxicity may also reduce the drug's therapeutic efficacy. This is especially true for the aminoglycosides since their therapeutic index is narrower than that observed with $\beta$-lactam antibiotics, for example. Although some members of the aminoglycoside family have been available since the early days of antibiotic therapy, it is only during the last decade that new insights have been provided into the pharmacodynamic activity of these drugs. Those new insights are the focus of discussion in this review.

\section{Efficacy}

The time-honoured method of assessing the antibacterial activity of the aminoglycosides is by measurement of the MIC and the MBC. Although these values are influenced by the concentration of divalent cations, the $\mathrm{pH}$ of the medium, and the inoculum (Reller et al., 1974; Thrupp, 1986), standardized techniques provide reasonable accuracy for these measurements. The activity of the aminoglycosides in human serum is virtually identical to that in broth with similar cation content, and this provides the rationale for using serum bactericidal activity as an indication of efficacy (Stratton \& Reller, 1977; Van, der Auwera \& Klastersky, 1987). Parameters such as the MIC and MBC give only a partial view of the full pharmacodynamic activity of these drugs. The MBC does not provide the bactericidal rate or show whether the latter is enhanced by increasing concentrations. Furthermore, the MIC does not reveal whether drugs exhibit persistent suppression of bacterial growth after antibiotic exposure, a phenomenon referred to as the postantibiotic effect or PAE (Vogelman \& Craig, 1985; Craig \& Gudmundsson, 1986). In fact, the rate of bactericidal activity with increasing concentrations and the presence or absence of a postantibiotic effect (PAE) better describe the time course of antimicrobial activity than MIC or MBC.

Aminoglycoside antibiotics differ from $\beta$-lactams in that their bactericidal activity is rapid, and concentration-dependent over a much wider range of concentrations. This has been shown to be so in vilro (Shah, Junghanns \& Stille, 1976; Van der Auwera \& Klastersky, 1987) as well as in vivo (Vogelman \& Craig, 1986; Flueckiger, Feller \& Gerber, 1986). On the other hand, MacArthur et al. (1984) have suggested that killing by aminoglycosides is concentration-dependent only during the first hour and that this stage is followed by a fixed limited bactericidal rate which is not enhanced by increasing concentrations. This may be so, but studies with repeated doses of aminoglycosides in neutropenic mice have demonstrated concentration-dependent killing for several hours at least (Gudmundsson, Turnidge \& Craig, 1982; Vogelman et al., 1988b).

Aminoglycoside antibiotics produce PAEs against staphylococci and various Gramnegative bacilli (Vogelman \& Craig, 1985; Craig \& Gudmundsson, 1986; Van der Auwera \& Klastersky, 1987). In general, the duration of the PAE increases with longer exposure times or higher drug concentrations up to a maximal response. Although the rapid and relatively complete bactericidal activity of aminoglycosides against many bacteria makes it difficult to quantitate a maximal response, PAEs of 1-3 h have consistently been observed in vitro, both in broth and in human serum. In vivo, even longer PAEs $(4-8 \mathrm{~h})$ have been observed with Staphylococcus aureus, Klebsiella pneumoniae, and Pseudomonas aeruginosa (Craig \& Gudmundsson, 1986; Vogelman et al., 1988a). These pharmacodynamic characteristics of the aminoglycosides, namely con- 
centration-dependent bactericidal activity and the presence of prolonged postantibiotic suppression of bacterial growth, provide the therapeutic rationale for less frequent dosing of these drugs than in current clinical practice. The administration of large intermittent doses would produce high peak levels which might maximize the rate of killing, and the PAE would maintain efficacy when serum and tissue levels fell below the minimal effective concentration. Blaser, Stone \& Zinner (1985) used an in-vitro model, simulating human pharmacokinetics, to examine the efficacy of several aminoglycoside dosage regimens against staphylococci and Gram-negative bacilli. When combined with ceftazidime, netilmicin was as effective in this model when given as a single daily dose as when administered in three daily doses that provided $50 \%$ more total dosage per day (Blaser et al., 1985). Emergence of resistance did not occur during the combination therapy. Netilmicin alone as a once-daily administration was also superior to either a continuous infusion or an 8-h regimen of the same total dose per $24 \mathrm{~h}$, in terms of faster killing and greater reduction of bacterial numbers. Regimens that did not provide peak levels of at least ten times the MIC were associated with the selection of resistant subpopulations (Blaser et al., 1987). The emergence of aminoglycoside-resistant subpopulations during therapy has also been observed in vivo in normal and neutropenic mice (Gerber et al., 1982; Pechère et al., 1986). These observations provide another reason for maximizing the peak level by intermittent dosing.

There are a variety of animal studies that have compared the efficacy of continuous infusion or frequent dosing with infrequent (as long as once daily) administration of the same amount of aminoglycoside. No difference in outcome was observed against $P$. aeruginosa in rabbit endocarditis (Powell et al., 1983), chronic pneumonia in rats (Powell et al., 1983), acute pneumonia in guinea pigs (Kapusnik et al., 1988), peritonitis in rats (Mordenti, Quintiliani \& Nightingale, 1985), and thigh infection in neutropenic mice (Gerber et al., 1983). Infrequent dosing was more effective than continuous infusion or frequent bolus administration in acute pseudomonas pneumonia in guinea pigs (Powell et al., 1983) and in staphylococcal endocarditis in rabbits, in combination with nafcillin (Kapusnik \& Sande, 1986). On the other hand, 12- and 24h dosages of tobramycin were less effective than 4 and 8-h dosing regimens in a klebsiella pneumonia model in mice (Pechère, Letarte \& Pechère, 1987). Similar findings were observed with $4 \mathrm{~h}$ and $24 \mathrm{~h}$ dosing of tobramycin in pseudomonas pneumonia in neutropenic guinea pigs (Kapusnik et al., 1988). Conclusions drawn from experimental studies focusing on dosage intervals are limited by the fact that in small animals the drugs are eliminated at a much faster rate than in man. In simulated human pharmacokinetics, in both normal and granulocytopenic mice, it was demonstrated that a simulation of once daily administration to man of netilmicin (in combination with $\beta$ lactam drugs) was superior or at least equal to a simulation of a thrice-daily regimen (Kozak et al., 1985; Gerber et al., 1986).

Four human trials have found no statistical difference in outcome between once daily and 8-hourly administration or continuous infusion (Powell et al., 1983; Nordstrŏm et al., 1985) and between continuous infusion and 6-hourly dosing (Feld et al., 1977; Feld et al., 1984). However, none of these animal and human studies was designed to correlate specific pharmacokinetic parameters with efficacy. Several clinical studies involving patients with Gram-negative bacillary infections have demonstrated that peak serum bactericidal titres of at least 8 (Klastersky, Meunier-Carpentier \& Prevost, 1977; Sculier \& Klastersky, 1984), or peak serum concentrations greater than $5 \mathrm{mg} / \mathrm{l}$ for gentamicin and tobramycin and greater than $20 \mathrm{mg} / \mathrm{l}$ for amikacin (Noone et al., 1974; 
Moore, Smith \& Lietman, 1984) were associated with a significantly improved clinical response. Since bactericidal titres will be strongly correlated with both antibiotic concentration and sensitivity of causative micro-organisms, they should be expected to correlate more strongly with clinical outcome than serum concentrations alone. Indeed, in a reanalysis of their data, Moore, Lietman \& Smith (1987) observed that the ratio of the peak concentration to the MIC was the major determinant of the clinical response to aminoglycoside therapy. At maximum peak concentration: MIC ratios of 6:1 or less the average response rate was less than $70 \%$, as opposed to a clinical response rate of approximately $90 \%$ at ratios of $8: 1$ or higher.

Since each of the studies mentioned above used a narrow range of doses, administered primarily at one dosing frequency, it cannot be determined whether the peak drug level is the critical parameter or whether a higher peak concentration simply reflects a larger area under the concentration-versus-time curve (AUC) and persistence of inhibitory or bactericidal levels for a greater proportion of the dosage interval. By using a large number of dosing regimens in the neutropenic mouse-thigh model, Vogelman et al. (1988b) were able to reduce much of the interdependence among pharmacokinetic parameters and determine the parameter that correlated best with efficacy. Their studies demonstrated that the AUC or the total dose, rather than the peak level or the duration of inhibitory serum levels, was the major determinant of the efficacy of aminoglycosides against Gram-negative bacilli. However, efficacy was reduced when the dosing interval was longer than the time period during which serum levels exceeded the MIC plus the duration of the postantibiotic effect, both as determined in vitro.

In conclusion, the pharmacodynamic characteristics of the aminoglycosides allow for equal efficacy with a variety of different dosing regimens. Although the AUC or total dose appears to be the primary determinant of efficacy, peak levels greater than ten times the MIC may be advantageous in preventing the selection of aminoglycoside resistant subpopulations. The studies cited provide support for a once-daily aminoglycoside dosing regimen, but there are still insufficient data on the effectiveness of such a regimen in human infections.

\section{Toxicity}

Although the toxicity of aminoglycosides is distinguished as nephrotoxicity on the one hand, and ototoxicity and vestibulotoxicity on the other, these two aspects are clearly interwoven, in so far as the risk for ototoxicity increases with impaired renal function. Insights into ototoxicity, and especially vestibulotoxicity, are still less developed than those into renal toxicity, mainly because the site of the toxic lesion is less accessible. Nephrotoxicity certainly limits the clinical utility of aminoglycosides (Lietman, 1985). Therefore, the identification of factors associated with a greater incidence of renal damage is critical. Potential risk factors can be classified as those related to the drug and its administration and those related to the clinical condition of the patient (Table I).

Aminoglycosides are highly polar drugs which cross biological membranes poorly or not at all, but they are filtered by the glomerulus. After entering the luminal fluid of the proximal renal tubule a small portion of the total filtered drug is reabsorbed and stored in the proximal tubular cells. After charge-mediated binding, the drug is taken up into the cell in small invaginations of the cell membrane, by a mechanism called "carrier mediated pinocytosis' (Collier, Lietman \& Mitch, 1979). Within one hour after injec- 
tion, the drug is translocated into apical cytoplasmic vacuoles (Silverblatt \& Kuehn, 1979). These endocytic vesicles fuse with lysosomes, sequestering the aminoglycosides inside those organelles in an unchanged form. Since pinocytosis is a continuing phenomenon aminoglycosides tend to accumulate extensively inside the lysosomes. The lysosomes have been identified as the first organelles to show alterations during aminoglycoside treatment (De Broe et al., 1984). Once trapped in the lysosomes of proximal tubular cells aminoglycosides inhibit lysosomal phospholipases and sphingomyelinase (Laurent et al., 1982; Giuliano et al., 1984), resulting in lysosomal phospholipidosis, with a non-specific accumulation of polar phospholipids in myeloid bodies. Once a threshold in cortical drug concentration is reached, the lysosomal phospholipidosis progresses and the overloaded lysosomes continue to swell even in the absence of any further drug administration. This may result in the loss of integrity of the restricting membranes of lysosomes and release of large amounts of aminoglycosides, lysosomal enzymes and phospholipids into the cytosol. The extralysosomal aminoglycosides can gain access to other organelles, disturbing their functional integrity which may lead to cell death (Tulkens et al., 1984).

The nephrotoxicity of aminoglycosides is determined by two major variables: (a) the intrinsic potential of the drug to damage subcellular structures and (b) the amount of drug accumulation in the renal cortex (Kaloyanides \& Pastoriza-Munoz, 1980).

Any factor that increases the renal uptake of aminoglycosides is a risk factor for nephrotoxicity. The duration of exposure of the proximal tubular cells to aminoglycosides is a critical factor since it determines the extent of drug uptake. Persistent exposure undoubtedly results in increased renal drug levels. Dosage regimen is another important determinant of the extent of cortical aminoglycoside concentrations. Experimental nephrotoxicity caused by gentamicin is more severe when the total daily dose is divided or given by continuous infusion than when it is given as a single bolus (Frame et al., 1977; Reiner, Bloxham \& Thompson, 1978; Powell et al., 1983). The reason for this may be found in the renal cortical uptake-storage kinetics of various aminoglycosides (Giuliano et al., 1986). In the rat, steady-state elevations of serum gentamicin and netilmicin are associated with a nonlinear increase in renal cortical levels, strongly

Table I. Potential risk factors for aminoglycoside nephrotoxicity (De Broe, 1985)

\begin{tabular}{ll}
\hline Drug-related & \multicolumn{1}{c}{ Patient related } \\
\hline $\begin{array}{l}\text { Dose } \\
\text { duration } \\
\text { dosage regimen }\end{array}$ & Age \\
$\begin{array}{l}\text { Prior aminoglycoside } \\
\text { treatment }\end{array}$ & $\begin{array}{l}\text { Prior renal insufficiency } \\
\text { Hepatic insufficiency }\end{array}$ \\
$\begin{array}{l}\text { Choice of drug } \\
\text { 'Critically ill' patient } \\
\text { Sodium-volume depletion }\end{array}$ \\
$\begin{array}{l}\text { Associated drugs } \\
\text { diuretics } \\
\text { cyclosporin } \\
\text { cisplatin } \\
\text { amphotericin }\end{array}$ & Other causes \\
\hline
\end{tabular}


suggesting saturable uptake while cortical uptake of tobramycin is linearly related to serum concentrations. These findings imply for gentamicin and netilmicin that the fraction of drug taken up by proximal tubule cells is higher when the same amount of drug is given by continuous infusion than by intermittent or single injections. Cortical uptake of tobramycin, in the rat, is independent of the dosage schedule, agreeing with the linear uptake kinetics of this aminoglycoside.

In humans, in a study in renal cancer patients who underwent nephrectomy, a critical effect of dosage schedule on renal uptake of gentamicin and netilmicin was found. In those patients a single injection resulted in $30 \%$ and $50 \%$ lower cortical drug concentrations of netilmicin and gentamicin, respectively, than the administration of the same amount of drug by a $24 \mathrm{~h}$ continuous infusion (Verpooten et al., 1989). Although an impressive number of comparative studies of aminoglycoside nephrotoxicity in humans are available (Whelton, 1985), it is difficult to draw firm conclusions from these studies because of the multiple clinical variables and because most of the studies failed to achieve critical methodological standards. Very often detection of clinical nephrotoxicity was based on proving decreased glomerular filtration in the individual patient. This led to high threshold values, resulting in underestimation of minor damage. In comparative clinical studies, statistical analysis of parameters of glomerular filtration (e.g., serum creatinine) in different groups of patients would be much more sensitive in detecting minor differences between different aminoglycosides. In a short-term prospective, controlled, randomized, comparative study in humans using clinical dosage regimens, gentamicin, tobramycin and netilmicin could not be distinguished on the basis of either tissue accumulation or early drug-induced cellular effects (De Broe et al., 1983). Advanced age has been suggested as a risk factor for aminoglycoside nephrotoxicity, as aging is accompanied by a decreased capacity in renal function and a marked decline of the regenerative response to drug-induced cell injury.

Pre-existing renal disease does not appear to be a risk factor for the induction of further renal impairment by aminoglycosides. Adjusting the dose or extending the interval between doses is not very difficult and can be accomplished reliably with the use of simple nomograms or with computer programs. Although monitoring of serum levels remains mandatory, gross deviations from desired plasma concentrations are rarely observed when dose adjustment procedures are being used. Since in most clinical trials this precaution has been taken, it may have contributed to the apparent lack of risk in renal failure patients. Moreover, it was shown experimentally that in rats with chronic renal failure less aminoglycoside is accumulated than in normal rats at comparable serum concentrations (Verpooten et al., 1986; Pattyn et al., 1988).

Finally, a prospective clinical study has shown that liver disease is associated with the development of nephrotoxicity. Hepatic insufficiency leading to intra-renal vasoconstriction, reduced renal blood flow and stimulation of the renin-angiotensin system is the proposed pathophysiological mechanism (Moore et al., 1984).

The assessment of ototoxicity by conventional means, in clinical studies on aminoglycoside treated patients as well as in clinical practice, appears clearly inadequate. Careful clinical monitoring of hearing and vestibular functions is mandatory, but irreversible anatomical damage may have occurred before symptoms appear. Moreover, problems may arise for the first time days or even weeks after the treatment has ended. Conventional audiograms also lack sensitivity, since they omit the higher frequencies, the first to be affected by aminoglycosides (Tange et al., 1982). Three new approaches have recently been introduced to improve early detection, but none is 
perfect. Brain stem evoked response audiometry (BERA) is a non-invasive, objective and sensitive method by which minute cochlear lesions might be recognized at a reversible stage (Pechère \& Bernard, 1982). As BERA requires no active participation from the patient, it can be used in neonates, infants and comatose individuals. Limitations of the method include relatively high costs for performing the test, the need for a reference recording in the individual patients before therapy and the multiple interferences of anoxia, neurological disorders, intubation, agitation of the patient, etc. Also, the actual significance of altered recordings at the anatomical and physiological level remains uncertain, so that interpretation is difficult, notably in neonates. Electrocochleography is another objective technique but the need to put an electrode through the ear drum limits its clinical application. High frequency tone audiometry is indeed very promising for patients who can actively respond to this subjective method (Dreschler et al., 1985; Tange, Dreschler \& Van der Hulst, 1985). Ototoxic alterations can be shown while the patient does not yet complain of hearing loss or tinnitus (Jacobson, Downs \& Fletcher, 1969). However, again reference testing has to be done before therapy when the patient is often too ill for reliable responses to be obtained. The same holds true for the methods available for monitoring the vestibular function.

The pharmacokinetics of aminoglycosides in the inner ear have recently been clarified. It was shown that the uptake of gentamicin, netilmicin and amikacin into different inner ear tissues did not correlate with the degree and the anatomical specificity of toxicity (Dulon et al., 1986), indicating a very specific cellular target (Aran et al., 1988). It was also observed that ototoxicity and nephrotoxicity induced by gentamicin did not correlate (Dulon et al., 1988a). Moreover, it was also demonstrated that cochlear damage induced by gentamicin was not associated with an accumulation of the drug into the inner ear (Tran Ba Huy, Bernard \& Schacht, 1986). The uptake of gentamicin by these tissues, however, is dose-dependent and manifests rapid saturation kinetics, followed by a concentration plateau and a very slow release. As a consequence, the prolonged exposure of the hair cells to the aminoglycoside probably accounts for the damage. Comparisons of different aminoglycosides with regard to their respective ototoxicity still poses problems, notably since the differences in nephrotoxicity are often not taken into account in animal studies. However, it has been shown experimentally that toxicity towards the cochlear and the vestibular portions may vary between drugs (Aran et al., 1982). Streptomycin is essentially vestibulotoxic, while amikacin is exclusively cochleotoxic. Gentamicin and tobramycin attack both targets, although the latter is somehwat less vestibulotoxic. Dibekacin has similar vestibulotoxicity to tobramycin, but exhibits little cochleotoxicity; netilmicin shows no cochleotoxicity and little vestibulotoxicity (Aran et al., 1982). The reasons for these quantitative differences remain unclear, as the structure of the two receptor organs is very similar, and the differential toxicity is not correlated with differential uptake of drug. In-vitro studies dealing with separate hair cells (Brownell et al., 1985; Zenner, Zimmermann \& Schmitt, 1985; Dulon et al., 1988b), as well as uptake studies at the cellular level using immunohistology and autoradiography, indicate indeed that the aminoglycosides interfere specifically with the hair cells (Hayashida et al., 1985; Aran et al., 1988).

Very few reports have addressed the issue of the ototoxicity with regard to dosage schedules. These studies are limited by ethical problems when conducted on patients, and by dosing problems when carried out in experimental animals. Dosages used in animals are multiples of those used in humans in terms of $\mathrm{mg} / \mathrm{kg}$, and even in terms of 
$\mathrm{mg} /$ body area, and hence are difficult to extrapolate to the clinical situation. In addition, different dosage schedules may have different impacts on renal function, which, in turn can impair the inner ear through the accumulation kinetics. These considerations might explain apparently conflicting experimental results. In rats monitored by brain stem evoked potentials, two or three daily doses appeared more vestibulo- and cochleotoxic than the same total dose given once a day (Pechère \& Bernard, 1984). Similar results were found in guinea pigs (Brummett \& Fox, 1982). In contrast, Davis et al. (1984), found experimentally that the magnitude of kanamycin ototoxicity was related to the total daily dose alone and not to the dosing schedule. With gentamicin, however, the dosage regimen does affect the uptake of drug by the inner ear. Less antibiotic penetrates into the cochlear tissues and fluids after a single intramuscular injection than after equal doses administered as a continuous infusion (Tran Ba Huy, Bernard \& Schacht, 1986). Thus, it may well be that wider dosing intervals produce less inner ear damage even though, in the latter study, the uptake of gentamicin was not directly correlated with toxicity. In patients with cystic fibrosis no differences with regard to ototoxicity were seen when the same daily dose of tobramycin was given for about ten days either by continuous infusion or by once daily dosing (Powell et al., 1983).

In humans, ototoxicity can be only poorly predicted from serum concentrations. Some older studies suggested a correlation between peak or trough serum aminoglycoside concentrations and ototoxicity. In a retrospective study on tuberculous patients, it was found that high trough levels of streptomycin predisposed to ototoxicity but those patients who had elevated trough levels were also older and had impaired renal function (Line, Poole \& Waterworth, 1970). Nordström et al. (1973) showed a significant correlation between high trough levels of gentamicin and ototoxicity, in a well conducted prospective study, but toxicity also correlated with high total dosage, longer duration of therapy and, once again, with abnormal renal function. Black et al. (1976), in monitoring for ototoxicity patients treated with amikacin, found that $57 \%$ of patients with a peak serum concentrations exceeding $32 \mathrm{mg} / 1$, and $55 \%$ of patients with trough concentrations exceeding $10 \mathrm{mg} / \mathrm{l}$, developed cochlear damage. However, the group with high frequency hearing loss received a larger mean total dose and were treated for a longer period than the patients without audiometric changes. It is sometimes suggested that the area under the time concentration curve, rather than peak or trough values might be a major risk factor for ear damage (Barza \& Lauermann, 1978), but there are no experimental or clinical studies substantiating this hypothesis. On the other hand, a specific threshold for peak or trough concentrations of aminoglycosides, above which ototoxicity occurs, can also not be identified: severe damage can occur in individuals with 'normal' serum concentrations and sometimes in a very unexpected and brutal fashion. The experimental and clinical studies with less frequent dosing schedules, in keeping the same daily dose, as described above, tend to obscure the correlations between serum levels and ototoxicity found previously. However, increased serum half-life, peak concentrations, trough concentrations or area under the time concentration curve may reflect impaired renal function, which is a recognized risk factor of ototoxicity.

In conclusion, there are experimental and clinical data that suggest that variation of dosage regimens may broaden the therapeutic index of aminoglycosides. Decreased dosage frequency may decrease nephro- as well as oto- and vestibulotoxicity of aminoglycosides, although tobramycin might be an exception to the rule. On the other 
hand experimental data suggest that efficacy is at least not decreased by increasing the dosage interval. Clinical data are still insufficient to draw strong conclusions, either about the equal efficacy of decreased dosage frequency, or about nephro- and ototoxicity. Part of the lack of clinical data on toxicity may be the result of inadequate use of available methods, including statistical sophistication, to make satisfactory use of the data. Future clinical trials should determine multiple pharmacokinetic parameters in patients (e.g., peak level, ratio of peak to MIC, AUC, AUC above MIC, and duration of inhibitory serum levels) so that multiple variate analysis can identify the major parameters determining efficacy. These studies should also look closely for resistant subpopulations by direct plating of clinical specimens on aminoglycoside-containing plates. They should also concentrate on the use of refined clinical methods to detect toxicity.

\section{Acknowledgements}

The workshop was made possible by the financial help of Lilly Nederland, Essex Nederland and Essex Benelux.

Editor-in-Chiefs note. This review will be complemented by a forthcoming leading article, which will discuss further clinical comparisons of dosage regimens, assess in terms of these the present evidence supporting less frequent dosage and define the cconomic benefit of reducing the dosage frequency.

\section{References}

Aran, J.-M., Dulon, D., Erre, J.-P., Hayashida, T. \& Hiel, H. (1988). Evidence that gentamicin acts directly on the hair cells of the cochlea. A physiopathological, immunohistochemical and radioautographic study in the guinea-pig. Journal of Physiology 406, 58P.

Aran, J.-M., Erre, J. P., Guilhaume, A. \& Aurousseau, C. (1982). The comparative ototoxicities of gentamicin, tobramycin and dibekacin in the guinea pig. A functional and morphological cochlear and vestibular study. Acta Otolaryngologica 390, Suppl., 1-30.

Barza, M. \& Lauermann, M. (1978). Why monitor serum levels of gentamicin? Clinical Pharmacokinetics 3, 202-15.

Black, R. E., Lau, W. K., Weinstein, R. J., Young, L. S. \& Hewitt, W. L. (1976). Ototoxicity of amikacin. Antimicrobial Agents and Chemotherapy 9, 956-61.

Blaser, J., Stone, B. B., Groner, M. C. \& Zinner, S. H. (1985). Impact of netilmicin regimens on the activities of ceftazidime-netilmicin combinations against Pseudomonas aeruginosa in an in vitro pharmacokinetic model. Antimicrobial Agents and Chemotherapy 28, 64-8.

Blaser, J., Stone, B. B., Groner, M. C. \& Zinner, S. H. (1987). Comparative study with enoxacin and netilmicin in a pharmacodynamic model to determine importance of ratio of antibiotic peak concentration to MIC for bactericidal activity and emergence of resistance. Antimicrobial Agents and Chemotherapy 31, $1054-60$.

Blaser, J., Stone, B. B. \& Zinner, S. H. (1985). Efficacy of intermittent versus continuous administration of netilmicin in a two-compartment in vitro model. Antimicrobial Agents and Chemotherapy 27, 343-9.

Brownell, W. E., Bader, C. R., Bertrand, D. \& de Ribaupierre, Y. (1985). Evoked mechanical responses of isolated cochlear outer hair cells. Science 227, 194-6.

Brummett, R. E. \& Fox, K. E. (1982). Studies of aminoglycoside ototoxicity in animal models. In The Aminoglycosides. Microbiology, Clinical use and Toxicology (Whelton, A. \& Neu, H. C., Eds), pp. 419-51. Marcel Dekker, New York.

Collier, V. U., Lietman, P. S. \& Mitch, W. E. (1979). Evidence for luminal uptake of gentamicin in the perfused rat kidney. Journal of Pharmacology and Experimental Therapeutics 210, 247-51. 
Craig, W. A. \& Gudmundsson, S. (1986). The postantibiotic effect. In Antibiotics in Laboratory Medicine, 2nd edn (Lorian, V., Ed.), pp. 515-36. Williams \& Wilkins, Baltimore, MD.

Davis, R. R., Brummeth, R. E., Bendrick, T. W. \& Himes, D. L. (1984). Dissociation of maximum concentration of kanamycin in plasma and perilymph from ototoxic effect. Journal of Antimicrobial Chemotherapy 14, 291-302.

De Broe, M. E. (1985). Prevention of aminoglycoside nephrotoxicity. In Proceedings of the European Dialysis and Transplant Association, Vol. 22 (Davison, A. M., Ed.), pp. 959-73. Baillière Tindall, London.

De Broe, M. E., Paulus, G. J., Verpooten, G. A. Giuliano, R. A., Roels, F. \& Tulkens, P. M. (1983). Early toxicity of aminoglycosides in human kidney: a prospective, comparative study of amikacin, gentamicin, netilmicin and tobramycin. In Proceedings of the 13th International Congress of Chemotherapy. Vienna, 1983, Vol. 9 (Spitzy, K. H. \& Karrer, K., Eds), SE 8.4/12, 86/1 1-24. Verlag H. Egermann, Vienna.

De Broe, M. E., Paulus, G. J., Verpooten, G. A., Roels, F., Buyssens, N., Wedeen, R. et al. (1984). Early effects of gentamicin, tobramycin, and amikacin on the human kidney. Kidney International 25, 643-52.

Dreschler, W. A., Van der Hulst, R. J. A. M., Tange, R. A. \& Urbanus, N. A. M. (1985). The role of high-frequency audiometry in the early detection of ototoxicity. Audiology 24, 38795.

Dulon, D., Aran, J.-M., Zajic, G. \& Schacht, J. (1986). Comparative uptake of gentamicin, netilmicin, and amikacin in the guinea pig cochlea and vestibule. Antimicrobial Agents and Chemotherapy 30, 96-100.

Dulon, D., Aurousseau, C., Erre, J.-P. \& Aran, J.-M. (1988a). Relationship between the nephrotoxicity and ototoxicity induced by gentamicin in the guinea pig. Acta Otolaryngologica 106, 219-25.

Dulon, D., Hayashida, T., Hiel, H., Erre, J. P. \& Aran, J. M. (1988b). Uptake of gentamicin and modifications to function of the sensory cochlear hair cells in vitro and in vivo. In vitro methods of pharmaco-toxicology, Paris 3-4 March 1988. Cytotechnology, Suppl. (June), 39.

Feld, R., Rachlis, A., Tuffnell, P. G., Duncan, I., Moran, L., Pinfold, P. et al. (1984). Empiric therapy for infections in patients with granulocytopenia. Continuous $v$ interrupted infusion of tobramycin plus cefamandole. Archives of Internal Medicine 144, 1005-10.

Feld, R., Valdivieso, M., Bodey, G. P. \& Rodriguez, V. (1977). A comparative trial of sisomicin therapy by intermittent versus continuous infusion. American Journal of the Medical Sciences 274, 179-88.

Flueckiger, U., Feller, C. \& Gerber, A. U. (1986). Dose-response curves in a man-adapted model: a novel view of comparative in vivo assessment of antibiotics. In Program and Abstracts of the Twenty-Sixth Interscience Conference on Antimicrobial Agents and Chemotherapy, New Orleans, LA 1986. Abstract 577, p. 202. American Society for Microbiology, Washington, DC.

Frame, P. T., Phair, J. P., Watanakunakorn, C. \& Bannister, T. W. P. (1977). Pharmacologic factors associated with gentamicin nephrotoxicity in rabbits. Journal of Infectious Diseases $135,952-6$.

Gerber, A. U., Brugger, H.-P., Feller, C., Stritzko, T. \& Stalder, B. (1986). Antibiotic therapy of infections due to Pseudomonas aeruginosa in normal and granulocytopenic mice: comparison of murine and human pharmacokinetics. Journal of Infectious Diseases 153, 90-7.

Gerber, A. U., Craig, W. A., Brugger, H.-P., Feller, C., Vastola, A. P. \& Brandel, J. (1983). Impact of dosing intervals on activity of gentamicin and ticarcillin against Pseudomonas aeruginosa in granulocytopenic mice. Journal of Infectious Diseases 147, 910-7.

Gerber, A. U., Vastola, A. P., Brandel, J. \& Crajg, W. A. (1982). Selection of aminoglycosideresistant variants of Pseudomonas aeruginosa in an in vivo model. Journal of Infectious Diseases 146, 691-7.

Giuliano, R. A., Paulus, G. J., Verpooten, G. A., Pattyn, V. M., Pollet, D. E., Nouwen, E. J. et al. (1984). Recovery of cortical phospholipidosis and necrosis after acute gentamicin loading in rats. Kidney International 26, 838-47.

Giuliano, R. A., Verpooten, G. A., Verbist, L., Wedeen, R. P. \& De Broe, M. E. (1986). In vivo uptake kinetics of aminoglycosides in the kidney cortex of rats. Journal of Pharmacology and Experimental Therapeutics 236, 470-5. 
Gudmundsson, S., Tumidge, J. \& Craig, W. A. (1982). Effect of different dosage regimens on in vivo efficacy of antibiotics against Klebsiella pneumoniae. Clinical Research 30, 777A.

Hayashida, T., Nomura, Y., Iwamori, M., Nagai, Y. \& Kurata, T. (1985). Distribution of gentamicin by immunofluorescence in the guinea pig inner ear. Archives of Otorhinalaryngology 242, 257-64.

Jacobson, E. J., Downs M. P. \& Fletcher, J. L. (1969). Clinical findings in high-frequency thresholds during ototoxic drug usage. Journal of Auditory Research 9, 379-85.

Kaloyanides, G. J. \& Pastoriza-Munoz, E. (1980). Aminoglycoside nephrotoxicity. Kidney International 18, 571-82.

Kapusnik, J. E., Hackbarth, C. J., Chambers, H. F., Carpenter, T. \& Sande, M. A. (1988). Single, large, daily dosing versus intermittent dosing of tobramycin for treating experimental pseudomonas pneumonia. Journal of Infectious Diseases 158, 7-12.

Kapusnik, J. E. \& Sande, M. A. (1986). Novel approaches for the use of aminoglycosides: the value of experimental models. Journal of Antimicrobial Chemotherapy 17, Suppl. A, 7-10.

Klastersky, J., Meunier-Carpentier, F. \& Prevost, J.-M. (1977). Significance of antimicrobial synergism for the outcome of gram negative sepsis. American Journal of the Medical Sciences 273, $157-67$.

Kozak, S., Gerber, A. U., Greter, U., Feller, C. \& Bangerter, T. (1985). Once daily vs. TIDdosing of netilmicin in combination therapy of $P$. aeruginosa in granulocytopenic mice. 14 th International Congress of Chemotherapy, Kyoto. Abstract S-85-8.

Laurent, G., Carlier, M. B., Rollman, B., Van Hoof, F. \& Tulkens, P. (1982). Mechanism of aminoglycoside-induced lysosomal phospholipidosis: in vitro and in vivo studies with gentamicin and amikacin. Biochemical Pharmacology 31, 3861-70.

Lietman, P. S. (1985). Aminoglycosides and spectinomycin: aminocyclitols. In Principles and Practice of Infectious Diseases, 2nd edn (Mandel, G. L., Douglas, R. G. \& Bennett, J. E., Eds), pp. 192-206. John Wiley, New York.

Line, D. H., Poole, G. W. \& Waterworth, P. M. (1970). Serum streptomycin levels and dizziness. Tubercle 51, 76-81.

MacArthur, R. D., Lolans, V., Zar, F. A. \& Jackson, G. G. (1984). Biphasic, concentrationdependent and rate-limited, concentration-independent bacterial killing by an aminoglycoside antibiotic. Journal of Infectious Diseases 150, 778-9.

Moore, R. D., Smith, C. R. \& Lietman, P. S. (1984). The association of aminoglycoside plasma levels with mortality in patients with gram-negative bacteremia. Journal of Infectious Diseases 149, 443-8.

Moore, R. D., Lietman, P. S. \& Smith, C. R. (1987). Clinical response to aminoglycoside therapy: importance of the ratio of peak concentration to minimal inhibitory concentration. Journal of Infectious Diseases 155, 93-9.

Mordenti, J. J., Quintiliani, R. \& Nightingale, C. H. (1985). Combination antibiotic therapy: comparison of constant infusion and intermittent bolus dosing in an experimental animal model. Journal of Antimicrobial Chemotherapy 15, Suppl. A, 313-21.

Noone, P., Parsons, T. M. C., Pattison, J. R., Slack, R. C. B., Garfield-Davies, D. \& Hughes, K. (1974). Experience in monitoring gentamicin therapy during treatment of serious gramnegative sepsis. British Medical Journal $i, 477-81$.

Nordström, L., Banck, G., Belfrage, S., Juhlin, I., Tjernström, Ö. \& Toremalm, N. G. (1973). Prospective study of the ototoxicity of gentamicin. Acta Pathologica et Microbiologica Scandinavica. Section B 81, Suppl. 241, 58-61.

Nordstrōm, L., Cronberg, S., Ringberg, H., Tjernström, Ö. \& Walder, M. (1985). Prospective, comparative, randomized clinical study of aminoglycosides given once a day versus three times a day in severe infections. In Recent Advances in Chemotherapy. Antimicrobial Section 3. Proceedings of the 14th International Congress of Chemotherapy, Kyoto, 1985 (Ishigami, J., Ed.), pp. 2653-4. University of Tokyo Press, Tokyo.

Pattyn, V. M., Verpooten, G. A., Giuliano, R. A. Zheng, F. \& De Broe, M. E. (1988). Effect of byperfiltration, proteinuria and diabetes mellitus on the uptake kinetics of gentamicin in the kidney cortex of rats. Journal of Pharmacology and Experimental Therapeutics 244, 694-8.

Pechère, J. C. \& Bernard, P. A. (1982). Surveillance des traitements par aminosides au moyen des potentiels evoqués du tronc cérébral. In Nephrotoxicité Ototoxicité Médicamenteuses (Fillastre, J. P., Ed.), pp. 345-57. Edition INSERM, Rouen, France. 
Pechère, J. C. \& Bernard, P. A. (1984). Gentamicin ototoxicity can be avoided if a new therapeutic regimen is used. An experimental animal model. In Program and Abstracts of the Twenty-Fourth Interscience Conference on Antimicrobial Agents and Chemotherapy, Washington, DC, 1984. Abstract 484, p. 178. American Society for Microbiology, Washington, DC.

Pechère, M., Letarte, R., \& Pechère, J.-C. (1987). Efficacy of different dosing schedules of tobramycin for treating a murine Klebsiella pneumoniae bronchopneumonia. Journal of Antimicrobial Chemotherapy 19, 487-91.

Pechère, J. C., Marchou, B., Michéa-Hamzehpour, M. \& Auckenthaler, R. (1986). Emergence of resistance after therapy with antibioties used alone or combined in a murine model. Journal of Antimicrobial Chemotherapy 17, Suppl. A, 11-8.

Powell, S. H., Thompson, W. L., Luthe, M. A., Stern, R. C., Grossniklaus, D. A., Bloxham, D. D. et al. (1983). Once-daily vs. continuous aminoglycoside dosing: efficacy and toxicity in animal and clinical studies of gentamicin, netilmicin, and tobramycin. Journal of Infectious Diseases 147, 918-32.

Reiner, N. E., Bloxham, D. D. \& Thompson, W. L. (1978). Nephrotoxicity of gentamicin and tobramycin given once daily or continuously in dogs. Journal of Antimicrobial Chemotherapy 4, Suppl. A, 85-101.

Reller, L. B., Schoenknecht, F. D., Kenny, M. A. \& Sherris, J. C. (1974). Antibiotic susceptibility testing of Pseudomonas aeruginosa: selection of a control strain and criteria for magnesium and calcium content in media. Journal of Infectious Diseases 130, 454-63.

Sculier, J. P. \& Klastersky, J. (1984). Significance of serum bactericidal activity in gram-negative bacillary bacteremia in patients with and without granulocytopenia. American Journal of Medicine 76, 429-35.

Shah, P. M., Junghanns, W. \& Stille, W. (1976). Dosis-Wirkungs-Beziehung der Bakterizidie bei E. coli, K. pneumoniae und Staphylococcus aureus. Deutsche Medizinische Wochenschrift 101, $325-8$.

Silverblatt, F. J. \& Kuehn, C. (1979). Autoradiography of gentamicin uptake by the rat proximal tubule cell. Kidney International 15, 335-45.

Stratton, C. W. \& Reller, L. B. (1977). Serum dilution test for bactericidal activity I. Selection of a physiologic diluent. Journal of Infectious Diseases 136, 187-95.

Tange, R. A., Conijn, E. A., Van Zeijl, L. G. \& Huizing, E. H. (1982). Pattern of gentamicininduced cochlear degeneration in the guinea pig. A morphological and electro-physiological study. Archives of Otorhinolaryngology 236, 173-84.

Tange, R. A., Dreschler, W. A. \& Van der Hulst, R. J. A. M. (1985). The importance of hightone audiometry in monitoring for ototoxicity. Archives of Otorhinolaryngology 242, 77-81.

Thrupp, L. D. (1986). Susceptibility testing of antibiotics in liquid media. In Antibiotics in Laboratory Medicine, 2nd edn (Lorian, V., Ed.), pp. 93-150. Williams \& Wilkins, Baltimore, MD.

Tran Ba Huy, P., Bernard, P. \& Schacht, J. (1986). Kinetics of gentamicin uptake and release in the rat. Comparison of inner ear tissues and fluids with other organs. Journal of Clinical Investigation 77, 1492-500.

Tulkens, P. M., De Broe, M. E., Maldague, P. \& Heuson-Stiennon, J. A. (1984). Lysosomal alterations in aminoglycoside-induced acute renal failure. In Acute Renal Failure Correlations between Morphology and Function (Solez, K. \& Whelton, A., Eds), pp. 299-327. Marcel Dekker, New York.

Van der Auwera, P. \& Klastersky, J. (1987). Serum bactericidal activity and postantibiotic effect in serum of patients with urinary tract infection receiving high-dose amikacin. Antimicrobial Agents and Chemotherapy 31, 1061-8.

Verpooten, G. A., Giuliano, R. A., Pattyn, V. M., Scharpé, S. L. \& De Broe, M. E. (1986). Renal cortical uptake kinetics of gentamicin in rats with impaired renal function. American Journal of Kidney Diseases 8, 304-7.

Verpooten, G. A., Giuliano, R. A., Verbist, L., Eestermans, G. \& De Broe, M. E. (1989). Oncedaily dosing decreases renal accumulation of gentamicin and netilmicin. Clinical Pharmacology and Therapeutics 45, 22-7.

Vogelman, B. S. \& Craig, W. A. (1985). Postantibiotic effects. Journal of Antimicrobial Chemotherapy 15, Suppl. A, 37-46. 
Vogelman, B. S. \& Craig, W. A. (1986). Kinetics of antimicrobial activity. Journal of Pediatrics 108, 835-40.

Vogelman, B., Gudmundsson, S., Tumidge, J., Leggett, J. \& Craig, W. A. (1988a). In vivo postantibiotic effect in a thigh infection in neutropenic mice. Journal of Infectious Diseases 157, 287-98.

Vogelman, B., Gudmundsson, S., Leggett, J., Turnidge, J., Ebert, S. \& Craig, W. A. (1988b). Correlation of antimicrobial pharmacokinetic parameters with therapeutic efficacy in an animal model. Journal of Infectious Diseases 158, $831-47$.

Whelton, A. (1985). Therapeutic initiatives for the avoidance of aminoglycoside toxicity. Journal of Clinical Pharmacology 25, 67-81.

Zenner, H. P., Zimmermann, U. \& Schmitt, U. (1985). Reversible contraction of isolated mammalian cochlear hair cells. Hearing Research 18, 127-33.

(Received 30 March 1989; accepted 12 May 1989) 